\title{
Vivre en communauté ou entre communautés ? Une réflexion sur le middle ground des États latins d'Orient
}

Florian Besson

\section{(2) OpenEdition \\ Journals}

Édition électronique

URL : http://journals.openedition.org/questes/4342

DOI : 10.4000/questes.4342

ISSN : 2109-9472

Éditeur

Les Amis de Questes

\section{Édition imprimée}

Date de publication : 6 mai 2016

Pagination : 35-50

ISSN : 2102-7188

\section{Référence électronique}

Florian Besson, «Vivre en communauté ou entre communautés?

Une réflexion sur le middle ground des États latins d'Orient », Questes [En ligne], 32 | 2016, mis en ligne

le 10 mai 2016, consulté le 19 avril 2019. URL : http://journals.openedition.org/questes/4342 ; DOI :

10.4000/questes.4342 


\title{
Vivre en communauté ou entre communautés? Une réflexion sur le middle ground des États latins d'Orient ${ }^{1}$
}

\author{
Florian BESSON \\ Université Paris-Sorbonne
}

$\mathrm{Au} \mathrm{XIX}^{\mathrm{e}}$ siècle, l'Académie des Inscriptions et Belles-Lettres entreprit de réunir et de publier tous les « historiens des croisades » dans des tomes volumineux qui couvrent l'ensemble de la période ${ }^{2}$. Ces tomes s'articulent comme suit: lois, historiens occidentaux, historiens orientaux, historiens grecs, historiens arméniens. Or c'est là un choix pour le moins contestable, qui correspond en fait à une vision du monde, et non à une décision scientifique. On aurait pu en effet imaginer d'autres modes de classement, notamment chronologique, ce qui aurait eu plus de sens d'un point de vue purement historique. Sans probablement s'en rendre compte, l'Académie contribuait par ce découpage à forger une vision des croisades qui domine encore très largement aujourd'hui : on insiste sur l'opposition entre des communautés bien structurées et clairement identifiées. Contrairement à ce qu'indiquent les titres, ce n'est pas l'origine géographique mais la religion qui est posée à la base de ces identités : un Guillaume de Tyr, né en Orient, archevêque de Tyr, parlant l'arabe, est évidemment placé dans les historiens «occidentaux»,

\footnotetext{
${ }^{1}$ Je tiens ici à remercier très sincèrement Rahul Markovits : c'est son séminaire à l'ENS de la rue d'Ulm en 2012-2013 qui m'a fait découvrir l'auteur qui est ici au cœur de ma réflexion. Merci aux Questeurs pour leurs remarques et questions et, comme toujours, à Catherine Kikuchi, pour la relecture et le soutien.

${ }^{2}$ Recueil des historiens des croisades (désormais abrégé en $R H C$ ), Paris, Imprimerie Royale, Impériale puis Nationale, 1841-1906, 16 vol.
} 
puisqu'il est chrétien. À l'inverse, Ibn Djubayr, musulman andalou, est classé dans les historiens orientaux : on comprend bien que «oriental» n'est qu'une façon polie de dire «musulman ». Ce découpage éditorial n'est donc pas innocent: il présuppose l'existence de communautés d'identités, regroupant des acteurs appartenant à divers milieux sociaux et à diverses époques, mais censés ainsi partager, en même temps qu'une religion, une même identité. En la présupposant, ce découpage contribuait aussi évidemment à la construire : le classement se faisait hiérarchie, et les sources arabes se voyaient reléguées en marge d'une histoire qui devait surtout s'écrire à partir des textes latins. Même si de telles perspectives ont été récemment critiquées ${ }^{3}$, elles restent très présentes ${ }^{4}$.

Quittons l'érudition du XIX ${ }^{\mathrm{e}}$ siècle pour revenir aux faits. Lorsque la première croisade s'achève en 1099, les Francs ont dû se retrouver dans une position très embarrassante : contrairement à certaines attentes ${ }^{5}$, la prise de Jérusalem n'avait pas entraîné la Parousie, le retour du Christ, et il fallait donc encore se battre, défendre les terres nouvellement conquises, et, surtout, régner, gouverner. Les Francs, très peu nombreux, ont donc dû gouverner un ensemble de territoires marqués par une grande diversité : diversité religieuse (catholiques, musulmans, mais aussi Grecs orthodoxes, juifs, et surtout chrétiens d'Orient avec toutes leurs différences), ethnique (Francs, Arabes, Kurdes, Turcs, Syriens, Arméniens) et sociale (des paysans aux nobles). Dès lors, pendant que les différents États latins se construisaient peu à peu, c'est la définition même

\footnotetext{
3 Voir par exemple Francesco Gabrieli, Chroniques arabes des croisades, Paris, Sindbad, coll. «Bibliothèque arabe », 1986.

4 Aamin Maalouf intitule son livre Les Croisades vues par les Arabes (Paris, J.C. Lattès, coll. "Histoire », 1984), comme si la croisade était l'élément central auquel «les Arabes», encore une communauté posée comme un présupposé, ne faisaient que réagir en la « voyant».

${ }^{5}$ Voir André Vauchez, «Les composantes eschatologiques de l'idée de croisade », dans Le Concile de Clermont de 1095 et l'appel à la croisade, Roma, École Française de Rome, 1997, p. 233-243.
} 
de la communauté qui est devenue un enjeu. Qu'est-ce qui faisait communauté, comment construire cette communauté, comment l'administrer au jour le jour? Derrière cette question, il y avait nombre d'enjeux très concrets : pouvait-on faire d'un musulman, par définition infidèle, son vassal ? Un contrat commercial conclu avec un musulman était-il valide ? Pouvait-on se confesser à un prêtre oriental, notamment lorsque l'on se trouvait en danger de mort ? Ces trois questions soulignent que c'est la communauté qui est en jeu, que ce soit sur le plan politique, économique, ou religieux.

En m'appuyant sur des exemples concrets, et en croisant sources occidentales et sources orientales, je me propose d'étudier la façon dont s'articulent ces communautés plurielles. Les groupes sont-ils séparés par des frontières ou bien reliés par des ponts et des passerelles qui autoriseraient à parler d'une vie en communauté ? Pour le dire autrement, comment conjuguer l'exigence d'unité, qui sous-tend l'idée même de communauté, et le contact, souvent sur le mode de la confrontation, avec l'autre ? Comment définir le commun alors qu'on est sans cesse confronté à l'altérité ?

\section{Un peu d'historiographie : de la colonie aux deux mondes}

Cette société marquée par la diversité a d'abord et surtout été lue comme une colonie. On insistait sur la domination politique des Occidentaux, ainsi que sur la très forte militarisation de la société. On a longtemps vu dans les croisades le début de l'expansion européenne, et les comparaisons étaient présentes dans tous les esprits depuis la fin du $\mathrm{XIX}^{\mathrm{e}}$ siècle $^{6}$. Cela ira en s'accentuant avec la colonisation, par exemple

\footnotetext{
${ }^{6}$ Emmanuel-Guillaume Rey, Les Colonies franques de Syrie aux XII et XIII siècles, Paris, Picard, 1883.
} 
chez René Grousset ${ }^{7}$, ou plus encore chez Joshua Prawer ${ }^{8}$, en écho avec la colonisation de la Palestine par Israël. Ce dernier notamment insistait sur le fait que les Francs avaient organisé un véritable «apartheid » qui les isolait des autres communautés. Assez vite critiqué, et aujourd'hui moins présent chez les historiens, ce paradigme de la colonie a été et continue à être utilisé fréquemment par les archéologues et historiens de l'art, prompts notamment à utiliser des termes comme «colonial» ou «métropole » sans forcément les interroger".

Or le modèle de la colonie est un mauvais modèle, qui ne recouvre que bien mal la réalité médiévale. L'implantation des Francs en SyriePalestine ne comporte ni exploitation économique généralisée, ni mise en valeur systématique du territoire, deux éléments structurants dans la définition de la colonisation. Surtout, c'est la domination politique qui pose problème: parler de l'Occident comme d'une métropole, c'est oublier que les États latins d'Orient sont des entités politiques autonomes, et que le roi de Jérusalem n'est pas un gouverneur chargé de veiller aux intérêts occidentaux en Terre sainte: c'est un souverain qui dirige son royaume. La colonie, que ce soit comme grille de lecture ou comme métaphore, doit donc être abandonnée.

Deuxième grille de lecture, plus tenace : le melting pot. Ce modèle s'est développé en partie en réaction au modèle précédent : on insistait

\footnotetext{
${ }^{7}$ René Grousset, Histoire des croisades et du royaume franc de Jérusalem, Paris, Plon, 1934-1936.

8 Joshua Prawer, The Latin Kingdom of Jerusalem. European colonialism in the Middle Ages, London, Weidenfeld and Nicolson, 1972; ou encore «Colonization Activities in the Latin Kingdom of Jerusalem », Revue belge de philologie et d'histoire, 1951, vol. 29, no 4, p. 1063-1118.

${ }^{9}$ Voir par exemple Alan M. Stahl, The Venetian Tornesello. A medieval colonial coinage, New York, The American Numismatic Society, 1985 ; La Fortification au temps des croisades, dir. Nicolas Faucherre, Jean Mesqui et Nicolas Prouteau, Rennes, PUR, coll. "Archéologie et culture », 2004 ; Jaroslav Folda, The Art of the crusaders in the Holy Land, 1098-1187, Cambridge, Cambridge University Press, 1995.
} 
dès lors sur l'autonomie des différents acteurs, sur la vigueur des échanges commerciaux, sur les transferts culturels ${ }^{10}$, etc. Plus que la domination des Latins, on soulignait l'assimilation culturelle et le métissage, ce qui participait aussi des encounter studies ${ }^{11}$. On mettait l'accent sur les influences mutuelles et les emprunts: les Francs découvrant les pigeons voyageurs et les bains, la canne à sucre et le feu grégeois, les Arabes empruntant aux Latins leurs cottes de maille et leur mot pour dire bourgeoisie. On parlait de «multiculturalisme ${ }^{12} »$. Dès lors, on pouvait commencer à penser une communauté qui ne soit plus basée uniquement sur l'identité géographique: les intermariages notamment, soulignés par Foucher de Chartres $^{13}$, contribuent à brouiller les frontières entre les groupes, tout comme les déplacements plus ou moins volontaires de populations. Cette vision du melting pot est intéressante, et elle a eu le grand mérite de déplacer l'intérêt des chercheurs des affrontements aux échanges. Mais elle reste politiquement connotée et gênante, car elle présuppose un résultat: si melting pot il y a, c'est qu'il doit y avoir fabrication d'une société mêlée. De plus, il reste alors la barrière

${ }^{10}$ Voir Jean Richard, Orient et Occident au Moyen Âge. Contacts et relations (XII ${ }^{e}$ $X V^{e}$ siècles), London, Variorum Reprints, 1976; plus encore, The Meeting of two worlds. Cultural exchange between East and West during the period of the crusades, dir. Vladimir P. Goss et Christine V. Bornstein, Kalamazoo, Medieval Institute Publications, 1986 ; le modèle informe aussi, quoique plus discrètement, l'ouvrage Outremer. Studies in the history of the crusading kingdom of Jerusalem, presented to Joshua Prawer, dir. Benjamin Z. Kedar, Hans E. Mayer et Raymond C. Smail, Jérusalem, Yad Izhak Ben-Zvi Institute, 1982, ou encore les nombreux ouvrages et articles de Michel Balard.

${ }^{11}$ Voir Serge Gruzinski, La Pensée métisse, Paris, Fayard, 1999.

${ }^{12}$ Un exemple parmi d'autres, Jaroslav Folda, «Crusader Art in the twelfth century. Reflections on Christian multiculturalism in the Levant », Mediterranean Historical Review, n $^{\text {10 }}$ 10, 1995, p. 80-91.

${ }^{13}$ Foucher de Chartres, Historia Hierosolymitana, dans RHC, Historiens occidentaux, tome III, Paris, Imprimerie impériale, 1866, cit. III, 37, p. 468 : «ille vero jam duxit uxorem non tamen compatriotam, sed et Syram aut Armenam, et interdum Sarracenam, baptismi autem gratiam adeptam », « tel autre d'entre nous a épousé une femme qui n'est point sa compatriote, une Syrienne ou une Arménienne, ou parfois même une Sarrasine, mais qui a reçu la grâce du baptême ». Nous traduisons. 
religieuse : on aurait l'image de Francs qui savent assimiler des chrétiens d'Orient mais qui restent clairement opposés aux musulmans.

Pour parler des relations entre Latins et musulmans, la figure qui s'imposait alors était, troisième et dernière grille de lecture, celle des « deux mondes », qui se seraient affrontés la plupart du temps et auraient réussi à échanger à l'occasion ${ }^{14}$. On trouvait en fait cette lecture depuis longtemps sous la forme «Orient/Occident ${ }^{15}$ ». Image séduisante par sa simplicité, mais qui justement tourne à la simplification abusive.

Car parler de «deux mondes », c'est simplifier à l'extrême la réalité : c'est présupposer l'existence de ces «mondes», de ces communautés. L'Orient latin n'est pas le théâtre d'un affrontement entre deux mondes, encore moins d'un choc des civilisations. S'y articulent en effet plusieurs mondes. Le « monde arabe », d'une part, est loin d'être un bloc: qu'on pense à la force des oppositions religieuses entre shi'ites, sunnites et ismaéliens, ou ethniques entre Arabes, Turcs et Kurdes. Le monde chrétien, d'autre part, n'est pas non plus d'une pièce: non seulement les divisions religieuses entre catholiques et chrétiens d'Orient restaient fortes, mais les Occidentaux eux-mêmes ne formaient pas un bloc. Il faut donc proposer une nouvelle grille de lecture, qui soit à même de rendre toute sa complexité à l'enchevêtrement des communautés (contre la théorie des deux mondes), sans présupposer pour autant un mouvement qui irait vers une assimilation à terme (contre la théorie du melting pot).

14 Citons par exemple The Meeting of two xorlds, op. cit.; Jean Richard, «Affrontement ou confrontation? Les contacts entre deux mondes au Pays de Tripoli au temps des croisades ", Chronos. Revue d'histoire de l'université de Balamand, $\mathrm{n}^{\mathrm{o}} 2,1999$, p. 7-28.

15 Depuis au moins Claude Cahen, Orient et Occident au temps des croisades [1983], Paris, Flammarion, coll. « Collection historique », 2010 ; citons aussi par exemple Les Croisades. L'Orient et l'Occident d'Urbain II à saint Louis, 1096-1270, dir. Monique Rey-Delque, Milano, Electra, 1997 ; Occident et Proche-Orient. Contacts scientifiques au temps des croisades, dir. Isabelle Draelants, Anne Tihon et Baudouin van den Abeele, Turnhout, Brepols, coll. «Réminisciences », 2000. 


\section{Le Middle Ground de l'Orient latin}

Cette nouvelle grille de lecture, je propose de la construire autour d'un paradigme élaboré par Richard White pour parler de la région des Grands Lacs entre le XVII ${ }^{\mathrm{e}}$ et le XIX siècle $^{16}$ : le Middle Ground. Ce terme, qu'on ne traduit pas mais qui pourrait se traduire par « entremonde » ou «monde commun », désigne un terrain d'adaptation culturelle commune. Les différents acteurs (en l'occurrence, chez White, les Français, les Anglais et les diverses tribus indiennes du Canada), ne pouvant se passer les uns des autres, et ne pouvant imposer leurs normes par la force, sont contraints d'inventer un langage diplomatique commun qui leur permette de développer des échanges commerciaux et même une alliance politico-militaire. Pour le dire concrètement, le Middle Ground, c'est le fait que le gouverneur du Canada couvre les chefs indiens de cadeaux, fume le calumet de la paix, et construise l'autorité française non pas sur la domination militaire mais sur la médiation dans les conflits entre Indiens. Le concept repose sur une réévaluation de la notion d'identité, articulée autour des travaux de James Clifford ${ }^{17}$. Le livre a eu beaucoup d'échos, mais curieusement le concept inscrit au centre de l'ouvrage a été assez peu testé pour d'autres époques, alors même que White le pose clairement comme un outil de travail, une création qui n'est pas spécifique à cette période ou à ce lieu, distinguant entre le Middle Ground, celui dont il parle, et un middle ground, qu'on peut identifier ailleurs et à d'autres époques. Or je pense que ce concept s'applique assez

\footnotetext{
${ }^{16}$ Richard White, Le Middle Ground. Indiens, empires et républiques dans la région des Grands Lacs, 1650-1815, Toulouse, Anacharsis, coll. « Essais », 2009.

${ }^{17}$ Citation placée en exergue de l'introduction de R. White : James Clifford, Malaise dans la culture. L'ethnographie, la littérature et l'art au $X X^{e}$ siècle, Paris, École nationale supérieure des Beaux-Arts, coll. «Espaces de l'art», 1996, cit. p. 23 : «Que se passe-t-il lorsque l'identité est conçue non comme [une] frontière mais comme un réseau de relations et de transactions dans lequel un sujet est activement impliqué ? L'histoire ou les histoires de l'interaction doivent alors se faire plus complexes, moins linéaires et moins téléologiques ».
} 
bien à l'Orient latin et peut nous permettre de repenser la façon dont se construisent et s'articulent les communautés.

Quelques semaines avant la prise de Jérusalem, Godefroy de Bouillon, duc de Basse-Lotharingie et futur premier souverain de la Ville sainte, était entré en relations avec l'émir d'Hasarth, Omar. Celui-ci avait confié au duc son fils comme otage, pratique courante tant en Occident qu'en Orient. Or voilà que ce jeune homme meurt d'une fièvre, mettant de fait Godefroy dans une position très délicate: son père risque d'accuser le duc de sa mort et de lui retirer son alliance. Voilà ce que fait alors le duc selon le chroniqueur allemand Albert d'Aix :

Après l'avoir fait envelopper d'une pourpre précieuse, selon l'usage des Gentils, le duc le renvoya à son père, lui déclarant, en toute bonne foi, qu'il n'avait aucune négligence à se reprocher à l'occasion de la mort de cet enfant, et protestant en être aussi vivement affecté qu'il eût pu l'être s'il eût perdu son frère Baudouin. Le prince reçut avec bonté les excuses du duc [...] il ne renonça point à la foi qu'il avait jurée, et demeura fermement attaché à l'alliance et à l'accord conclus par lui avec le duc Godefroi ${ }^{18}$.

Autrement dit, à un moment difficile, Godefroy, ayant besoin de l'émir, sait s'adapter aux exigences de ce dernier : il utilise «l'usage des Gentils »(morem gentilium), autrement dit une grammaire politique orientale. On voit que la grille de lecture du melting pot ne fonctionne pas: il ne s'agit pas d'une acculturation, d'une orientalisation de Godefroy. Le chef franc n'était pas non plus un colon imposant ses

18 Albert d'Aix, Liber christianae expeditionis pro ereptione, emundatione, restitutione sanctae Hierosolymitanae ecclesiae, dans $R H C$, Historiens occidentaux, t. IV, Paris, Imprimerie Nationale, 1879, p. 265-713, cit. V, 32, p. 450 : «Quem juxta morem gentilium pretioso ostro involutum patri remisit, excusans se sub omni fidei puritate, quod ex causa suae negligentiae minime puer obiisset, obtestans etiam se non minus ejus mortem quam fratris sui Baldewini moleste ferre. Qui ducis excusatione benigne accepta, et ex vero comperta ab his, quos de domo sua custodes pueri misit, nequaquam immutatus est a fide quam promisit, sed immobilis in omni foedere et pace cum ipso duce et fratre ejus Baldewino permansit. ». Nous traduisons. 
normes ou acceptant celles de l'« indigène » avec condescendance. Au contraire, Godefroy choisit consciemment d'utiliser des gestes et des pratiques propres à une autre communauté.

Cette adaptation culturelle et politique suppose un intérêt intellectuel pour l'autre, une curiosité, une ouverture à l'autre. Celle-ci est souvent déniée aux croisés, et, de fait, elle est assez peu présente dans les sources. Mais a-t-on assez tenu compte du fait que ces sources étaient écrites pour l'Occident, par des clercs, et qu'il s'agissait donc probablement d'insister sur l'opposition, de renforcer l'antagonisme, afin d'attirer de nouveaux chevaliers impatients de venir combattre l'infidèle et d'obtenir, ce faisant, le salut de leurs âmes ? Au quotidien, la réalité devait être bien différente, et nombre de témoignages discrets attestent de la multiplicité des échanges. En frappant des monnaies à légendes arabes $^{19}$, en acceptant de commercer avec des musulmans, en maintenant certaines mosquées ouvertes dans les villes conquises, les Francs firent le choix de la relation, privilégiant la possibilité d'un échange avec l'autre, aussi limité soit-il, plutôt que l'isolation et l'enfermement sur euxmêmes.

\section{Le choix du lien}

De fait, alors que la question de la communauté ne cessait de se poser, les chroniques latines attestent d'un décalage : décalage entre les constructions savantes des clercs qui racontent l'histoire et les choix pragmatiques de ceux qui la vivent et la font. Pour la majorité des clercs, il était probablement impossible de faire d'un musulman un vassal : le musulman était un païen, donc un infidèle, par définition impossible à intégrer dans des relations de fidélité. L'homonymie entre la foi et la

\footnotetext{
${ }^{19}$ Paul Balog et Jacques Yvon, «Monnaies à légende arabe de l'Orient latin », Revue numismatique, $1958,6^{\text {ème }}$ série, $\mathrm{n}^{\mathrm{o}} 1$, p. 133-168.
} 
fidélité, toutes deux exprimées par le même terme de fides, servait ici à exclure l'autre : le musulman ne pouvait donc être qu'un traître, un faux fidèle, attendant la moindre occasion pour poignarder dans le dos son naïf seigneur. Les choix des acteurs étaient beaucoup plus pragmatiques : dès le début, les croisés ont cherché des alliances parmi les musulmans, et n'ont jamais hésité à en conclure lorsqu'ils étaient sollicités. Ainsi de Godefroy, qui, on l'a vu, n'hésite pas à faire de l'émir d'Hasarth son vassal. Albert d'Aix emploie significativement, sans hésiter, le même terme de fidei pour le duc et pour le Turc : l'infidèle peut partager la fides du croisé. L'exemple est ici intéressant car il montre bien comment se construit le middle ground de l'Orient latin : le chef turc a appris le nom de Godefroy par une captive, femme d'un chevalier lorrain qu'il a faite prisonnière quelque temps auparavant. Il a besoin de son soutien face à un ennemi commun, en l'occurrence Ridwān d'Alep. Prudent, il envoie au duc un chrétien d'Orient, un « homme né chrétien et syrien ${ }^{20}$ » : en effet, ceux-ci ont très souvent été des émissaires tout trouvés, proches des Francs par leur religion, des musulmans par la langue et la culture, aptes à jouer les intermédiaires, comme les métis dans le Middle Ground de White. L'émir envoie son fils en otage pour rassurer Godefroy. Celui-ci, en retour, lui fait des cadeaux, lui offrant notamment un casque et une cuirasse $^{21}$. Les cadeaux jouent un rôle très important dans le middle ground: en échangeant des objets, on crée du lien social ${ }^{22}$.

Mais le middle ground n'est pas fait uniquement de cadeaux échangés. En 1155, Baudouin II de Jérusalem accorde ainsi son saufconduit à la famille d'Usāma ibn Munqidh, résidant alors en Égypte, mais

\footnotetext{
${ }^{20}$ Albert d'Aix, op. cit., V, 7, p. 437.

${ }^{21}$ Ibid., V, 12, p. 440.

${ }^{22}$ Voir, évidemment, Marcel Mauss, Essai sur le don. Forme et raison de l'échange dans les sociétés archaïques, Paris, PUF, coll. «Quadriges », 2012. Voir aussi Maurice Godelier, L'Énigme du don, Paris, Fayard, 1997. 
provoque ensuite le naufrage du navire qui la transporte, afin de pouvoir piller le bateau et rançonner les passagers. Un fidèle d'Usāma nage jusqu'au roi pour lui montrer son sauf-conduit, et celui-ci répond : «mais, nous suivons en ce moment l'usage des Musulmans : lorsqu'une de leurs embarcations fait naufrage en face d'un pays, les habitants de celui-ci ont le droit d'y exercer le pillage ${ }^{23} »$. Autrement dit, le roi de Jérusalem sait, lorsque cela l'arrange, utiliser une pratique musulmane, et surtout sait arguer, non sans cynisme, qu'il suit leur usage et qu'il n'est donc pas en tort. L'argumentation ici déployée renvoie directement à l'un des grands éléments du modèle de Richard White, la nécessité de justifier son action en empruntant le vocabulaire de l'autre ${ }^{24}$.

On voit donc qu'on a bien affaire à un middle ground, à cet effort volontaire et conscient pour construire un monde commun au sein duquel l'échange est possible. Il s'agit bien d'accommodements, maître mot de l'analyse de White: faute de pouvoir imposer ses pratiques, on «fait avec » celles de l'autre.

\section{Franchir les frontières, dessiner les limites}

Ce monde commun, fait de compromis, d'improvisations et d'accommodements, se construit dans un Orient latin très complexe : comme le rappelle bien Thomas Asbridge dans un article capital, les croisés n'édifient pas leur monde sur une table rase, mais sur un ensemble de communautés multiculturelles, multiconfessionnelles, multi-

\footnotetext{
${ }^{23}$ Usâma ibn Munqidh, Des Enseignements de la vie. Souvenirs d'un gentilhomme syrien du temps des Croisades, traduction française André Miquel, Paris, Imprimerie Nationale, 1983, cit. p. 187.

${ }^{24}$ Richard White, op. cit., p. 98 : «l'aspect crucial et déterminant du Middle Ground fut peut-être la volonté de ceux qui le créèrent de justifier leurs propres agissements dans les termes de ce qu'ils pensaient être les implicites culturels de leurs partenaires. »
} 
ethniques $^{25}$. Il faut essayer de saisir la grande diversité des acteurs, qui n'avaient pas forcément les mêmes buts : du seul côté de «l'Occident» se croisent ordres militaires, roi de Jérusalem, clergé, républiques marchandes italiennes, pape, seigneurs féodaux, etc. Comme l'écrit Richard White pour le Pays d'en Haut, «qualifier d'alliance cet imbroglio d'ambitions et d'objectifs, c'est comme considérer que les occupants d'un bordel une nuit donnée constituent une famille ${ }^{26} \gg$. Loin de voir se dessiner ainsi deux blocs, on se trouve face à un grand nombre de frontières, qui peuvent se superposer ou se croiser. Ces frontières sont de plus très poreuses : pensons à ces combats opposant des Francs et des musulmans des deux côtés. En 1109, par exemple, Tancrède et Ridwân d'Alep affrontèrent Djawâlî de Mossoul et Baudouin d'Édesse : des deux côtés du champ de bataille, il y avait donc des chevaliers francs luttant aux côtés de musulmans. Franchir ces frontières n'était pas l'œuvre de marginaux qui se seraient dès lors vus rejetés en marge de toutes les communautés: le Livre au Roi, un texte juridique datant de la fin du $\mathrm{XII}^{\mathrm{e}}$ siècle, autorise au contraire un chevalier franc à aller servir dans une armée musulmane, «en terre de Sarasins », s'il ne renie pas sa foi et s'il confie son fief au roi, fief qu'il peut même récupérer s'il revient avant un an et un jour ${ }^{27}$.

À travers ces frontières poreuses, les contacts personnels étaient possibles : Usāma ibn Munqidh peut parler des Templiers comme de ses amis. L'une de ses anecdotes est célèbre : alors qu'il prie à Jérusalem, un

\footnotetext{
25 Thomas S. Asbridge, «The "Crusader" community at Antioch. The impact of interaction with Byzantium and Islam », Transactions of the royal historical society, 1999, Sixth Series, vol. 9, p. 305-325, cit. p. 306. Asbridge va jusqu'à parler d'interdépendance des différentes communautés : je resterai, au moins pour l'instant, plus prudent.

${ }^{26}$ Richard White, op. cit., p. 321.

${ }^{27}$ Le Livre au Roi, éd. Myriam Greilsammer, Paris, Académie des Inscriptions et Belles-Lettres, coll. «Documents pour servir à l'histoire des croisades », 1995, ici ch. 22, p. 200-202.
} 
Franc se précipite vers lui et lui tourne la tête vers l'Orient en disant «c'est ainsi que l'on prie ${ }^{28} »$. Les Templiers, véritable police des Lieux Saints, expulsent violemment cet homme, qui vient d'arriver d'Occident et n'a probablement jamais vu quelqu'un prier en se tournant vers $\mathrm{La}$ Mecque et non vers l'est, et ils s'excusent auprès d'Usāma pour sa brutalité : la création et l'entretien du middle ground sont ici conscients, volontaires. Il faut du temps pour s'y habituer, et Usāma écrit ainsi : « tous les nouveaux arrivés en terre franque ont un comportement plus inhumain que ceux qui s'y sont acclimatés et ont fréquenté les musulmans ${ }^{29}{ }^{2}$. Il s'agit bel et bien ainsi de s'acclimater, d'apprendre le langage de ce monde commun, et les Francs nouvellement arrivés en Syrie ont généralement du mal à s'y insérer, commettant des bourdes et des impairs.

Plusieurs lois structurent ce middle ground, sur lesquelles il faudrait se pencher plus en profondeur : que l'on pense par exemple au phénomène de la trêve, généralement respectée par les parties en présence, ou encore à l'importance des échanges de prisonniers. Une autre des lois de ce middle ground était le respect et la protection pour les pèlerins: très vite en effet, les seigneurs francs placent sous leur protection les pèlerins qui vont à la Mecque. En retour, Tancrède, alors régent d'Antioche, envoie aux Banū Munqidh un chevalier «qui a fait le pèlerinage et va retourner chez lui », en leur demandant de «bien le traiter $^{30} »$. Loin de n'être qu'un élément divisant les communautés, la religion est dans ce cas au contraire un point commun, un lien, presque un pont, qui dessine des rapprochements et des convergences et autorise le

\footnotetext{
${ }^{28}$ Usāma ibn Munqidh, op . cit. p. 297.

29 Ibid. Même expression p. 303. On retrouve exactement la même chose chez Richard White, par exemple avec le général De Montcalm qui tente de traiter les guerriers indiens comme des soldats français, p. 348.

${ }^{30}$ Usāma ibn Munqidh, op. cit., p. 191.
} 
partage fragile d'une identité commune, celle de pèlerin, qui l'emporte temporairement sur les autres. Un autre point commun partageable est la position sociale : entre les nobles musulmans et chrétiens, des codes sont partagés, notamment le culte de l'honneur et du courage ${ }^{31}$, et ces convergences peuvent aller jusqu'à l'amitié : l'amitié de Roger d'Antioche avec le seigneur turc Tughtekin était célèbre, tout comme celle de Renaud de Sidon avec Saladin.

À tout seigneur tout honneur : j’ai commencé avec Godefroy, je finirai avec Saladin. Faisant preuve de clémence, respectant sa parole et en particulier les trêves, accessible aux chrétiens comme aux musulmans ${ }^{32}$, Saladin se caractérise par sa grande habileté à manœuvrer dans le middle ground, à en utiliser les codes, alors même qu'il réunissait les forces de l'Islam pour en finir avec la présence franque en Terre Sainte. Son intégration dans l'imaginaire chevaleresque occidental n'est peut-être, au fond, que la consécration ultime de cette habileté à vivre dans un espace social commun. Après son écrasante victoire à Hattin en 1187, Saladin a fait capturer le roi Guy de Jérusalem : pour rassurer son captif, qui craignait de se voir exécuter, Saladin déclare « les rois ne font pas mourir des rois $^{33} \gg$. Cela prouve l'existence de règles, de pratiques communes, qui font penser en l'occurrence à un jeu d'échecs. Ce jeu, d'ailleurs, partagé entre les différentes communautés, est une belle métaphore de ce middle ground: un terrain de jeu structuré par des règles qui autorisent l'échange, même si c'est toujours sur fond de conflit et d'affrontement. Le middle ground, ainsi, ne s'oppose pas à la violence,

\footnotetext{
${ }^{31}$ Voir Anne-Marie Eddé, «L'honneur des chevaliers francs dans les sources arabes à l'époque des croisades », dans L'Islam au carrefour des civilisations médiévales, dir. Michel Sot et Dominique Barthélémy, Paris, PUPS, coll. «Cultures et civilisations médiévales », 2012, p. 135-153.

32 Anne-Marie Eddé, Saladin, Paris, Flammarion, coll. « Grandes biographies », 2008, p. 321-367.

${ }^{33}$ Bahā ad-Dīn, Anecdotes et beaux traits de la vie du sultan Yûsuf, dans RHC, Historiens orientaux, tome III, Paris, Imprimerie Nationale, 1884, p. 3 370, cit. p. 97. 
il l'encadre: les pratiques partagées ne sont pas des parenthèses au milieu d'un océan d'incompréhension mais bien les symboles les plus évidents de l'existence d'un espace social commun.

Ainsi, il n'y a pas seulement deux communautés qui s'opposeraient en tout, mais une pluralité de groupes, sans cesse en recomposition. En particulier, il n'est pas sûr que le critère religieux ait toujours été le plus déterminant et soit forcément le mieux indiqué pour situer les acteurs : on a vu l'importance de l'appartenance à un même milieu social par exemple, et on peut rappeler que les enjeux politiques et géopolitiques l'emportaient le plus souvent sur les différences religieuses. Les Francs ont su sans problème intégrer les différentes minorités confessionnelles : plutôt que d'avoir en tête le modèle d'un royaume de France «très chrétien », il faudrait peut-être davantage comparer les États Latins d'Orient aux sociétés des marges de l'Occident chrétien, comme la péninsule ibérique, ou encore la Hongrie ${ }^{34}$. À travers leurs interactions, ces groupes dessinent une communauté à l'échelle de la région, faite de négociations et d'accommodements, toujours fragiles et éphémères. Plutôt que de parler en termes de contacts, d'affrontements ou d'échanges entre communautés, je propose d'envisager plutôt cette histoire en termes de création d'un monde commun.

Cette vision est très probablement à la fois trop optimiste et trop naïve, péchant notamment par irénisme, et il ne faut pas oublier les violences, tant réelles que symboliques. Les anciennes grilles de lecture étaient politiquement connotées, renvoyant à un imaginaire du fait colonial ou de l'assimilation sur le modèle américain. Le middle ground est lui aussi un modèle influencé par les tensions et les enjeux de notre

\footnotetext{
34 Voir Nora Berend, At the Gate of christendom. Jews, muslims and "pagan" in medieval Hungary, Cambridge, Cambridge University Press, 2006.
} 
temps. Comme l'écrit très bien Catherine Desbarats dans la préface du livre de White, «si cet acte d'imagination historique peut encore nous entraîner, c'est en partie parce qu'il est au diapason de notre sens contemporain du savoir, de la justice, et de ce que c'est que d'avoir été humain à travers les siècles ${ }^{35}{ }$.

${ }^{35}$ Catherine Desbarats, avant-propos de R. White, op. cit., p. 6. 